\title{
Exact Evaluation of Infinite Series Using Double Laplace Transform Technique
}

\author{
Hassan Eltayeb, ${ }^{1}$ Adem Kılıçman, ${ }^{2}$ and Said Mesloub ${ }^{1}$ \\ ${ }^{1}$ Mathematics Department, College of Science, King Saud University, P.O. Box 2455, Riyadh 11451, Saudi Arabia \\ ${ }^{2}$ Department of Mathematics and Institute for Mathematical Research, University Putra Malaysia, \\ 43400 Serdang, Selangor, Malaysia
}

Correspondence should be addressed to Adem Kılıçman; akilic@upm.edu.my

Received 10 October 2013; Accepted 28 December 2013; Published 20 January 2014

Academic Editor: S. A. Mohiuddine

Copyright (C) 2014 Hassan Eltayeb et al. This is an open access article distributed under the Creative Commons Attribution License, which permits unrestricted use, distribution, and reproduction in any medium, provided the original work is properly cited.

Double Laplace transform method was applied to evaluate the exact value of double infinite series. Further we generalize the current existing methods and provide some examples to illustrate and verify that the present method is a more general technique.

Finding the exact value of infinite series is not an easy task. Thus, it is a common way to estimate by using the certain test or methods. It is also known that there are certain conditions to apply the estimation methods. For example, some methods are only applicable for the series having only positive terms. However, there is no general method to estimate values of all the type series. One of the most valuable approaches to summing certain infinite series is the use of Laplace transforms in conjunction with the geometric series.

Efthimiou presented a method [1] that uses the Laplace transform and allows one to find exact values for a large class of convergent series of rational terms. Lesko and Smith [2] revisited the method and demonstrated an extension of the original idea to additional infinite series. Sofo [3] used a forced differential difference equation and by the use of Laplace Transform Theory generated nonhypergeometric type series. Dacunha, in [4], introduced a finite series representation of the matrix exponential using the Laplace transform for time scales. In [5], infinite series and complex numbers were applied to derive formulas. Abate and Whitt in their paper [6] applied infinite series and Laplace transform in study of probability density function by numerical inversion; see [7] for application to summing series arising from integrodifferential difference equations; also see Eltayeb in [8] who studied the relation between double differential transform and double Laplace transform by using power series. Our intention in this note is to illustrate the power of the technique in the case of double series.

The infinite series, whose summand $Q(n, m)=Q_{n} Q_{m}$ which is given by $\sum_{n=1}^{\infty} \sum_{m=1}^{\infty} Q_{n} Q_{m}$, can be realized as double Laplace transform as

$$
Q_{n} Q_{m}=\iint_{0}^{\infty} e^{-n t-m x} f(x) f(t) d t d x .
$$

In such a case, what appeared to be a sum of numbers is now written as a sum of integrals. This may not seem like progress, but interchanging the order of summation and integration yields a sum that we can evaluate easily, namely, the way of a geometric series consider

$$
\begin{aligned}
\sum_{n=1}^{\infty} \sum_{m=1}^{\infty} Q_{n} Q_{m} & \\
& =\int_{0}^{\infty} f(x) \sum_{m=1}^{\infty}\left(e^{-x}\right)^{m} d x \int_{0}^{\infty} f(t) \sum_{n=1}^{\infty}\left(e^{-t}\right)^{n} d t \\
& =\int_{0}^{\infty} f(x)\left(\frac{e^{-x}}{1-e^{-x}}\right) d x \int_{0}^{\infty} f(t)\left(\frac{e^{-t}}{1-e^{-t}}\right) d t
\end{aligned}
$$

We first illustrate the method for a special case. We then describe the general result pointing out further generalizations of the method, and we finally end with a brief discussion. 
Consider the series

$$
Q(\alpha, \beta ; c, d)=\sum_{n=1}^{\infty} \sum_{m=1}^{\infty} \frac{1}{(m+\alpha)(m+\beta)} \frac{1}{(n+c)(n+d)},
$$

where $\alpha \neq \beta$ and $c \neq d$ and neither $\alpha$ nor $\beta$ is a negative integer and also $c, d$. Sums of this form arise often in problems dealing with Quantum Field Theory. By using partial fractions, we have

$$
\begin{aligned}
Q(\alpha, \beta ; c, d)= & \frac{1}{\alpha-\beta} \sum_{m=1}^{\infty}\left(\frac{1}{(m+\beta)}-\frac{1}{(m+\alpha)}\right) \\
& \cdot \frac{1}{c-d} \sum_{n=1}^{\infty}\left(\frac{1}{(n+d)}-\frac{1}{(n+c)}\right) .
\end{aligned}
$$

By using double Laplace transform of function $f(t, x)=1$,

$$
\iint_{0}^{\infty} e^{-A t-B x} d t d x=\frac{1}{A B}, \quad A, B>0 .
$$

Therefore, for $\alpha, \beta>-1$ and $c, d>-1$, we have

$$
\begin{aligned}
Q(\alpha, \beta ; c, d) & \left(\frac{1}{(\alpha-\beta)} \lim _{M \rightarrow \infty} \sum_{m=1}^{M} \int_{0}^{\infty} e^{-m t}\left(e^{-\beta t}-e^{-\alpha t}\right) d t\right) \\
& \times\left(\frac{1}{(c-d)} \lim _{N \rightarrow \infty} \sum_{m=1}^{N} \int_{0}^{\infty} e^{-m x}\left(e^{-d x}-e^{-c x}\right) d x\right) \\
= & \left(\lim _{M \rightarrow \infty} \int_{0}^{\infty}\left(\frac{e^{-\beta t}-e^{-\alpha t}}{\alpha-\beta}\right) e^{-t} \frac{\left(1-e^{-M t}\right)}{1-e^{-t}} d t\right) \\
& \times\left(\lim _{N \rightarrow \infty} \int_{0}^{\infty}\left(\frac{e^{-d x}-e^{-c x}}{c-d}\right) e^{-x} \frac{\left(1-e^{-N x}\right)}{1-e^{-x}} d x\right) \\
= & \left(\int_{0}^{\infty}\left(\frac{e^{-\beta t}-e^{-\alpha t}}{\alpha-\beta}\right) \frac{e^{-t}}{1-e^{-t}} d t\right) \\
& \times\left(\int_{0}^{\infty}\left(\frac{e^{-d x}-e^{-c x}}{c-d}\right) \frac{e^{-x}}{1-e^{-x}} d x\right) .
\end{aligned}
$$

Using change of variables $y=e^{-t}$ and $z=e^{-x}$ in the integral of (6) gives a more symmetric result:

$$
Q(\alpha, \beta ; c, d)=\frac{1}{(\alpha-\beta)(c-d)} \iint_{0}^{1} \frac{y^{\beta}-y^{\alpha}}{1-y} \frac{z^{d}-z^{c}}{1-z} d y d z .
$$

The integral in (7) converges for $\alpha \neq \beta, c \neq d$, and $\alpha, \beta>-1$, $c, d>-1$. In case $\alpha=1 / 2, c=1 / 2$, and $\beta=0, d=0$, (7) becomes

$$
\begin{aligned}
\sum_{n=1}^{\infty} & \sum_{m=1}^{\infty} \frac{1}{m n(m+1 / 2)} \frac{1}{(n+1 / 2)} \\
& =4 \iint_{0}^{1} \frac{1-\sqrt{y}}{1-y} \frac{1-\sqrt{z}}{1-z} d y d z \\
& =4 \iint_{0}^{1} \frac{1}{(1+\sqrt{y})(1+\sqrt{z})} d y d z .
\end{aligned}
$$

By using substitution method, we have

$$
\sum_{n=1}^{\infty} \sum_{m=1}^{\infty} \frac{1}{m n(m+(1 / 2))} \frac{1}{(n+(1 / 2))}=(4(1-\ln 2))^{2} .
$$

For another example, take $\alpha=0, c=0$, and $\beta, d$ as positive integers; then (7) becomes

$$
\begin{aligned}
& \sum_{n=1}^{\infty} \sum_{m=1}^{\infty} \frac{1}{m(m+\beta)} \frac{1}{n(n+d)} \\
& =\frac{1}{\beta d} \iint_{0}^{1} \frac{1-y^{\beta}}{1-y} \frac{1-z^{d}}{1-z} d y d z \\
& =\frac{1}{\beta d} \iint_{0}^{1}\left(1+y+y^{2}+\cdots+y^{\beta-1}\right) \\
& \quad \times\left(1+z+z^{2}+\cdots+z^{d-1}\right) d y d z \\
& =\frac{1}{\beta d}\left(1+\frac{1}{2}+\frac{1}{3}+\cdots+\frac{1}{\beta}\right)\left(1+\frac{1}{2}+\frac{1}{3}+\cdots+\frac{1}{d}\right) .
\end{aligned}
$$

Example 1. Find closed-form expressions for series of the form

$$
\sum_{m=2}^{\infty} \frac{1}{\left(m^{2}-1\right)} \sum_{n=2}^{\infty} \frac{1}{\left(n^{2}-1\right)}
$$

We observe from the definition of double Laplace transform that

$$
\frac{1}{\left(p^{2}-1\right)\left(s^{2}-1\right)}=\iint_{0}^{\infty} e^{-s t-p x} \sinh (t) \sinh (x) d t d x .
$$

The hyperbolic sine of $t$ and $x$ can be expressed exponentially as follows:

$$
\sinh t=\frac{e^{t}}{2}-\frac{e^{-t}}{2}, \quad \sinh x=\frac{e^{x}}{2}-\frac{e^{-x}}{2} .
$$

Then (12) can be written as

$$
\begin{aligned}
& \frac{1}{\left(p^{2}-1\right)\left(s^{2}-1\right)} \\
& =\left(\frac{1}{2} \int_{0}^{\infty} e^{t} e^{-s t} d t-\frac{1}{2} \int_{0}^{\infty} e^{-t} e^{-s t} d t\right) \\
& \quad \times\left(\frac{1}{2} \int_{0}^{\infty} e^{x} e^{-p x} d x-\frac{1}{2} \int_{0}^{\infty} e^{-x} e^{-p x} d x\right)
\end{aligned}
$$

By using the general summation for (14), this becomes

$$
\begin{aligned}
\sum_{s=2}^{\infty} \frac{1}{\left(s^{2}-1\right)} \sum_{p=2}^{\infty} \frac{1}{\left(p^{2}-1\right)} \\
=\left(\frac{1}{2} \int_{0}^{\infty} e^{t} \sum_{s=2}^{\infty}\left(e^{-t}\right)^{s} d t-\frac{1}{2} \int_{0}^{\infty} e^{-t} \sum_{s=2}^{\infty}\left(e^{-t}\right)^{s} d t\right) \\
\quad \times\left(\frac{1}{2} \int_{0}^{\infty} e^{x} \sum_{s=2}^{\infty}\left(e^{-x}\right)^{p} d x-\frac{1}{2} \int_{0}^{\infty} e^{-x} \sum_{s=2}^{\infty}\left(e^{-x}\right)^{p} d x\right) .
\end{aligned}
$$


Let us make substitution of variables $y=e^{-x}$ and $z=e^{-t}$; we have

$$
\begin{aligned}
\sum_{s=2}^{\infty} & \frac{1}{\left(s^{2}-1\right)} \sum_{p=2}^{\infty} \frac{1}{\left(p^{2}-1\right)} \\
= & \left(\frac{1}{2} \int_{1}^{0} \frac{1}{z} \frac{-z^{2} d z}{(1-z) z}-\frac{1}{2} \int_{1}^{0} \frac{-z^{2} z d z}{(1-z) z}\right) \\
& \times\left(\frac{1}{2} \int_{1}^{0} \frac{1}{y} \frac{-y^{2} d y}{(1-y) y}-\frac{1}{2} \int_{1}^{0} \frac{-y^{2} y d y}{(1-y) y}\right) .
\end{aligned}
$$

Simplification of the above equation yields

$$
\begin{aligned}
& \sum_{s=2}^{\infty} \frac{1}{\left(s^{2}-1\right)} \sum_{p=2}^{\infty} \frac{1}{\left(p^{2}-1\right)} \\
& \quad=\left(\frac{1}{2} \int_{0}^{1}(1+z) d z\right)\left(\frac{1}{2} \int_{0}^{1}(1+y) d y\right)=\frac{9}{16} .
\end{aligned}
$$

In the next example we use the same method.

Example 2. As a variation, let us choose a general term with analog function of $s$ and $p$. A typical function of this type might be

$$
F(m, n)=\frac{16 m n}{\left(m^{2}-1\right)^{2}\left(n^{2}-1\right)^{2}} .
$$

It is desired to evaluate this series from $m=2, n=2$ to $m=$ $\infty, n=\infty$; by using table of Laplace transform, we have

$$
\begin{aligned}
& \frac{4 p}{\left(p^{2}-1\right)^{2}} \frac{4 s}{\left(s^{2}-1\right)^{2}} \\
& \quad=4 \iint_{0}^{\infty} e^{-s t-p x} x t \sinh (x) \sinh (t) d t d x .
\end{aligned}
$$

When the two numbers $\alpha, \beta$ and $c, d$ differ by an integer $k, r$, that is, $\alpha=\beta+k$ and $c=d+r$, respectively, then the sum of (3) can be easily calculated from (4):

$$
Q(\alpha, \alpha-k ; c, c-r)=\frac{1}{k r} \sum_{j=1}^{-k} \sum_{i=1}^{-r}\left(\frac{1}{j+\alpha}\right)\left(\frac{1}{i+c}\right) .
$$

The previous equation can be checked from (7) as follows:

$$
\begin{aligned}
Q(\alpha, \alpha-k ; c, c-r) & =\frac{1}{k r} \iint_{0}^{1} \frac{y^{k}-1}{y-1} y^{\alpha} \frac{z^{r}-1}{z-1} z^{c} d y d z \\
& =\frac{1}{k r} \iint_{0}^{1-k-1-r-1} \sum_{h=1} \sum_{l=1}^{h+\alpha} y^{l+c} d y d z \\
& =\frac{1}{k} \sum_{h=1}^{k-1}\left(\frac { y ^ { h + \alpha + 1 } } { h + \alpha + 1 } | _ { 0 } ^ { 1 } \frac { 1 } { r } \sum _ { l = 1 } ^ { r - 1 } \left(\left.\frac{z^{l+c+1}}{l+c+1}\right|_{0} ^{1} .\right.\right.
\end{aligned}
$$

Then

$$
Q(\alpha, \alpha-k ; c, c-r)=\frac{1}{k r} \sum_{j=1}^{-k} \sum_{i=1}^{-r}\left(\frac{1}{j+\alpha}\right)\left(\frac{1}{i+c}\right) .
$$

We now generalize Efthirniou's technique to the series of the form

$$
\sum_{n=1}^{\infty} \sum_{m=1}^{\infty} Q_{n} P_{n} u_{m} v_{m}
$$

where it is convenient to write that only

$$
Q_{n}=\int_{0}^{\infty} e^{-n t} f(t) d t, \quad u_{m}=\int_{0}^{\infty} e^{-m x} f(x) d x
$$

are Laplace transform as follows:

$$
\begin{aligned}
\sum_{n=1}^{\infty} Q_{n} P_{n} \sum_{m=1}^{\infty} u_{m} v_{m} \\
\quad=\int_{0}^{\infty} f(t)\left(\sum_{n=1}^{\infty} P_{n} e^{-n t}\right) d t \int_{0}^{\infty} f(x)\left(\sum_{m=1}^{\infty} v_{m} e^{-m x}\right) d x .
\end{aligned}
$$

For example, consider

$$
\sum_{n=1}^{\infty} \sum_{m=1}^{\infty} \frac{r^{n}}{\alpha n+\beta} \frac{k^{m}}{a m+b}
$$

where $r \in[-1,1), k \in[-1,1), \alpha, a>0, \beta, b \geq 0$.

By using Laplace transform

$$
\iint_{0}^{\infty} e^{-n t-m x}\left(e^{-(\beta / \alpha) t} e^{-(b / a) x}\right) \frac{d x d t}{\alpha a},
$$

where $r \neq-1, k \neq-1$, the partial sum of

$$
\sum_{n=1}^{\infty} \sum_{m=1}^{\infty} r^{n} k^{m} e^{-n t-m x}\left(e^{-(\beta / \alpha) t} e^{-(b / a) x}\right) \frac{1}{\alpha a}
$$

is dominated above by

$$
\begin{aligned}
\sum_{n=1}^{\infty}\left|r^{n} e^{-n t}\left(\frac{1}{\alpha} e^{-(\beta / \alpha) t}\right)\right| \sum_{m=1}^{\infty}\left|k^{m} e^{-m x}\left(\frac{1}{a} e^{-(b / a) x}\right)\right| \\
=\frac{1}{\alpha} e^{-(\beta / \alpha) t}\left(\frac{|r| e^{-t}}{1-|r| e^{-t}}\right) \frac{1}{a} e^{-(b / a) x} \\
\quad \times\left(\frac{|k| e^{-x}}{1-|k| e^{-x}}\right),
\end{aligned}
$$

where

$$
\begin{aligned}
& \int_{0}^{\infty} \frac{1}{\alpha} e^{-(\beta / \alpha) t}\left(\frac{|r| e^{-t}}{1-|r| e^{-t}}\right) d t \int_{0}^{\infty} \frac{1}{a} e^{-(b / a) x}\left(\frac{|k| e^{-x}}{1-|k| e^{-x}}\right) d x \\
& \quad \leq \frac{1}{\alpha a} \int_{0}^{\infty}\left(\frac{|r| e^{-t}}{1-|r| e^{-t}}\right) d t \\
& \quad \times \int_{0}^{\infty}\left(\frac{|k| e^{-x}}{1-|k| e^{-x}}\right) d x<\infty .
\end{aligned}
$$


We apply the Lebesgue dominated convergence theorem to have

$$
\begin{aligned}
& \sum_{n=1}^{\infty} \sum_{m=1}^{\infty} \frac{r^{n}}{\alpha n+\beta} \frac{k^{m}}{a m+b} \\
& =\sum_{n=1}^{\infty} \int_{0}^{\infty} r^{n} e^{-n t}\left(\frac{1}{\alpha} e^{-(\beta / \alpha) t}\right) d t \sum_{m=1}^{\infty} \int_{0}^{\infty} k^{m} e^{-m x} \\
& \quad \times\left(\frac{1}{a} e^{-(b / a) x}\right) d x \\
& =\int_{0}^{\infty}\left(\frac{1}{\alpha} e^{-(\beta / \alpha) t}\right) \sum_{n=1}^{\infty} r^{n} e^{-n t} d t \int_{0}^{\infty}\left(\frac{1}{a} e^{-(b / a) x}\right) \\
& =\frac{1}{\alpha a} \int_{0}^{\infty} e^{-(\beta / \alpha) t}\left(\frac{r e^{-t}}{1-r e^{-t}}\right) d t \int_{0}^{\infty} k^{m} e^{-m x} d x \\
& =\frac{1}{\alpha a} \iint_{0}^{1} \frac{r u^{\beta / \alpha}}{1-r u} \frac{k v^{b / a}}{1-k v} d u d v . \\
& =\left(\frac{k e^{-x}}{1-k e^{-x}}\right) d x
\end{aligned}
$$

Now, let $a=1, b=0$ and $\alpha=1, \beta=0$ yield

$$
\begin{aligned}
\sum_{n=1}^{\infty} \sum_{m=1}^{\infty} \frac{r^{n}}{n} \frac{k^{m}}{m} & =\iint_{0}^{1} \frac{r}{1-r u} \frac{k}{1-k v} d u d v \\
& =\ln \left(\frac{1}{1-r}\right) \ln \left(\frac{1}{1-k}\right)
\end{aligned}
$$

and when $r=-1, k=-1, a=1, \alpha=1$, and $b=1 / 2, \beta=1 / 2$, we have

$$
\begin{aligned}
\sum_{n=1}^{\infty} \sum_{m=1}^{\infty} \frac{(-1)^{n}}{n+(1 / 2)} \frac{(-1)^{m}}{m+(1 / 2)} & =\iint_{0}^{1} \frac{u^{1 / 2}}{1+u} \frac{v^{1 / 2}}{1+v} d u d v \\
& =\left(\frac{\pi}{2}-2\right)^{2} .
\end{aligned}
$$

Consider one more example of this technique

$$
\begin{aligned}
Q(\alpha, \beta ; a, b)= & \sum_{n=1}^{\infty} \sum_{m=1}^{\infty} \frac{k^{m}}{(m+a)(m+b)} \frac{r^{n}}{(n+\alpha)(n+\beta)} \\
= & \left(\sum_{n=1}^{\infty} r^{n} \int_{0}^{\infty} e^{-n t}\left(\frac{e^{-\alpha t}-e^{-\beta t}}{\beta-\alpha}\right) d t\right) \\
& \times\left(\sum_{m=1}^{\infty} k^{m} \int_{0}^{\infty} e^{-m x}\left(\frac{e^{-a x}-e^{-b x}}{b-a}\right) d x\right) \\
= & \left(\int_{0}^{\infty}\left(\frac{e^{-\alpha t}-e^{-\beta t}}{\beta-\alpha}\right) \sum_{n=1}^{\infty} r^{n} e^{-n t} d t\right)
\end{aligned}
$$

$$
\begin{aligned}
& \times\left(\int_{0}^{\infty}\left(\frac{e^{-a x}-e^{-b x}}{b-a}\right) \sum_{m=1}^{\infty} k^{m} e^{-m x} d x\right) \\
= & \left(\frac{1}{\beta-\alpha} \int_{0}^{\infty}\left(e^{-\alpha t}-e^{-\beta t}\right)\left(\frac{r e^{-t}}{1-r e^{-t}}\right) d t\right) \\
& \times\left(\frac{1}{b-a} \int_{0}^{\infty}\left(e^{-a x}-e^{-b x}\right)\left(\frac{k e^{-x}}{1-k e^{-x}}\right) d x\right) \\
= & \frac{r k}{(\beta-\alpha)(b-a)} \iint_{0}^{1} \frac{u^{\alpha}-u^{\beta}}{1-r u} \frac{v^{a}-v^{b}}{1-k v} d u d v .
\end{aligned}
$$

Now, let $a=1, b=0$ and $\alpha=1, \beta=0$, and $r, k \in(-1,1)$; then (34) becomes

$$
\begin{aligned}
& \sum_{n=1}^{\infty} \sum_{m=1}^{\infty} \frac{k^{m}}{m(m+1)} \frac{r^{n}}{n(n+1)} \\
& \quad=r k \iint_{0}^{1} \frac{1-u}{1-r u} \frac{1-v}{1-k v} d u d v \\
& \quad=\left(1+\left(\frac{1-r}{r}\right) \ln (1-r)\right)\left(1+\left(\frac{1-k}{k}\right) \ln (1-k)\right) .
\end{aligned}
$$

To apply the method to trigonometric series, we need to be able to handle series of the form

$$
\begin{aligned}
& S=\sum_{n=1}^{\infty} \sum_{m=1}^{\infty} \sin (m y) \sin (n z) e^{-m x-n t}, \\
& C=\sum_{n=1}^{\infty} \sum_{m=1}^{\infty} \cos (m y) \cos (n z) e^{-m x-n t} .
\end{aligned}
$$

In particular, we assume $y$ and $z$ are real number and $x>$ 0 and $t>0$, where

$$
\begin{aligned}
& \sum_{n=1}^{\infty} \sum_{m=1}^{\infty} \sin (m y) \sin (n z) e^{-m x-n t} \\
& \quad=\frac{e^{-x} \sin y}{1-2 \cos y e^{-x}+e^{-2 x}} \frac{e^{-t} \sin z}{1-2 \cos z e^{-t}+e^{-2 t}} \\
& \sum_{n=1}^{\infty} \sum_{m=1}^{\infty} \cos (m y) \cos (n z) e^{-m x-n t} \\
& \quad=\frac{e^{-x}\left(\cos y-e^{-x}\right)}{1-2 \cos y e^{-x}+e^{-2 x}} \frac{e^{-t}\left(\cos z-e^{-t}\right)}{1-2 \cos z e^{-t}+e^{-2 t}} .
\end{aligned}
$$

We start by the series

$$
\sum_{n=1}^{\infty} \sum_{m=1}^{\infty} \frac{\cos (n z) \cos (m y)}{n^{\nu} m^{\mu}}
$$

where $\nu, \mu \in \mathbb{N}$, and $0<y, z<2 \pi$ if $\mu, \nu=1$ or $0 \leq y, z \leq 2 \pi$ if $\mu, v>1$. Using

$$
\frac{1}{n^{\nu} m^{\mu}}=\frac{1}{(\nu-1) !(\mu-1) !} \iint_{0}^{\infty} e^{-m x-n t} t^{\nu-1} x^{\mu-1} d t d x
$$


we can write (38) as

$$
\begin{aligned}
\sum_{n=1}^{\infty} \sum_{m=1}^{\infty} \frac{\cos (n z) \cos (m y)}{n^{\nu} m^{\mu}} \\
=\left(\frac{1}{(\nu-1) !} \sum_{n=1}^{\infty} \cos (n z) \int_{0}^{\infty} e^{-n t} t^{\nu-1} d t\right) \\
\quad \times\left(\frac{1}{(\mu-1) !} \sum_{m=1}^{\infty} \cos (m y) \int_{0}^{\infty} e^{-m x} x^{\mu-1} d x\right) \\
=\left(\frac{1}{(\nu-1) !} \int_{0}^{\infty}\left(\sum_{n=1}^{\infty} \cos (n z) e^{-n t}\right) t^{\nu-1} d t\right) \\
\quad \times\left(\frac{1}{(\mu-1) !} \int_{0}^{\infty}\left(\sum_{m=1}^{\infty} \cos (m y) e^{-m x}\right) x^{\mu-1} d x\right) \\
=\left(\frac{1}{(\nu-1) !} \int_{0}^{\infty} \frac{e^{-t}\left(\cos z-e^{-t}\right)}{1-2 \cos z e^{-t}+e^{-2 t}} t^{\nu-1} d t\right) \\
\quad \times \frac{1}{(\mu-1) !} \int_{0}^{\infty} \frac{e^{-x}\left(\cos y-e^{-x}\right)}{1-2 \cos y e^{-x}+e^{-2 x}} x^{\mu-1} d x .
\end{aligned}
$$

With the change of variables $u=e^{-t}$ and $v=e^{-x}$ becomes

$$
\begin{aligned}
& \sum_{n=1}^{\infty} \sum_{m=1}^{\infty} \frac{\cos (n z) \cos (m y)}{n^{\nu} m^{\mu}} \\
& =\left(\frac{(-1)^{\nu-1}}{(\nu-1) !} \int_{0}^{1} \frac{(\cos z-u)}{1-2 \cos z u+u^{2}}(\ln u)^{\nu-1} d u\right) \\
& \quad \times \frac{(-1)^{\mu-1}}{(\mu-1) !} \int_{0}^{1} \frac{(\cos y-v)}{1-2 \cos y v+v^{2}}(\ln v)^{\mu-1} d v
\end{aligned}
$$

In the special case, let $\mu=1, \nu=1$; we have

$$
\begin{aligned}
\sum_{n=1}^{\infty} \sum_{m=1}^{\infty} \frac{\cos (n z) \cos (m y)}{n m} \\
=\left(-\frac{1}{2} \int_{0}^{1} \frac{d\left(1-2 \cos z u+u^{2}\right)}{1-2 \cos z u+u^{2}}\right) \\
\quad \times\left(-\frac{1}{2} \int_{0}^{1} \frac{d\left(1-2 \cos y v+v^{2}\right)}{1-2 \cos y v+v^{2}}\right) \\
=\left(-\frac{1}{2}\left[\ln \left(1-2 \cos z u+u^{2}\right)\right]_{0}^{1}\right) \\
\quad \times\left(-\frac{1}{2}\left[\ln \left(1-2 \cos y v+v^{2}\right)\right]_{0}^{1}\right) \\
=\ln \left(2 \sin \frac{z}{2}\right) \ln \left(2 \sin \frac{y}{2}\right) .
\end{aligned}
$$

We use the same steps for the series

$$
\sum_{n=1}^{\infty} \sum_{m=1}^{\infty} \frac{\sin (n z) \sin (m y)}{n^{\nu} m^{\mu}} ;
$$

we arrive at the integral representation

$$
\begin{aligned}
\sum_{n=1}^{\infty} \sum_{m=1}^{\infty} \frac{\sin (n z) \sin (m y)}{n^{\nu} m^{\mu}} \\
=\left(\frac{(-1)^{\nu-1}}{(\nu-1) !} \sin z \int_{0}^{1} \frac{(\ln u)^{\nu-1}}{1-2 \cos z u+u^{2}} d u\right) \\
\quad \times \frac{(-1)^{\mu-1}}{(\mu-1) !} \sin y \int_{0}^{1} \frac{(\ln v)^{\mu-1}}{1-2 \cos y v+v^{2}} d v
\end{aligned}
$$

In particular, for $\mu=1, \nu=1$,

$$
\begin{aligned}
\sum_{n=1}^{\infty} \sum_{m=1}^{\infty} \frac{\sin (n z) \sin (m y)}{n m} \\
=\left(\sin z \int_{0}^{1} \frac{1}{(u-\cos z)^{2}+\sin ^{2} z} d u\right) \\
\quad \times\left(\sin y \int_{0}^{1} \frac{1}{(v-\cos y)^{2}+\sin ^{2} y} d v\right) \\
\left.\left.=\mid \tan ^{-1} \frac{u-\cos z}{\sin z}\right]_{0}^{1} \mid \tan ^{-1} \frac{v-\cos y}{\sin y}\right]_{0}^{1} \\
=\left(\tan ^{-1} \frac{\sin z}{1-\cos z}\right)\left(\tan ^{-1} \frac{\sin y}{1-\cos y}\right) \\
=\left(\frac{\pi-y}{2}\right)\left(\frac{\pi-z}{2}\right) .
\end{aligned}
$$

\section{Conflict of Interests}

The authors declare that there is no conflict of interests regarding the publication of this paper.

\section{Acknowledgments}

The authors are grateful for the very useful comments regarding detailed remarks which improved the presentation and the contents of the paper. Further, the authors would also like to extend their sincere appreciation to the Deanship of Scientific Research at King Saud University for its funding of this research through the Research Group Project no. RGP-VPP117

\section{References}

[1] C. J. Efthimiou, "Finding exact values for infinite sums," Mathematics Magazine, vol. 72, no. 1, pp. 45-51, 1999.

[2] J. P. Lesko and W. D. Smith, "A Laplace transform technique for evaluating infinite series," Mathematics Magazine, vol. 76, no. 5, pp. 394-398, 2003.

[3] A. Sofo, "Summation of series via Laplace transforms," Revista Matemática Complutense, vol. 15, no. 2, pp. 437-447, 2002.

[4] J. J. Dacunha, "Transition matrix and generalized matrix exponential via the Peano-Baker series," Journal of Difference Equations and Applications, vol. 11, no. 15, pp. 1245-1264, 2005. 
[5] D. E. Dobbs, "Using infinite series and complex numbers to derive formulas involving Laplace transforms," International Journal of Mathematical Education in Science and Technology, vol. 44, no. 5, pp. 752-761, 2013.

[6] J. Abate and W. Whitt, "Infinite-series representations of Laplace transforms of probability density functions for numerical inversion," Journal of the Operations Research Society of Japan, vol. 42, no. 3, pp. 268-285, 1999.

[7] P. Cerone and A. Sofo, "Summing series arising from integrodifferential-difference equations," The Journal of Australian Mathematical Society B, vol. 41, no. 4, pp. 473-486, 2000.

[8] H. Eltayeb, "Note on relation between double Laplace transform and double differential transform," Abstract and Applied Analysis, vol. 2013, Article ID 535020, 7 pages, 2013. 


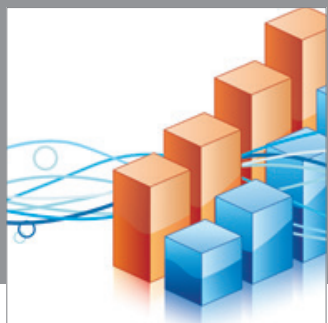

Advances in

Operations Research

mansans

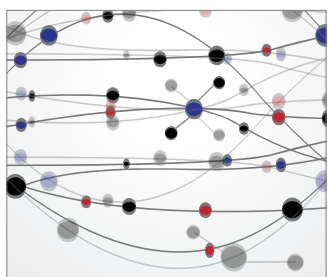

The Scientific World Journal
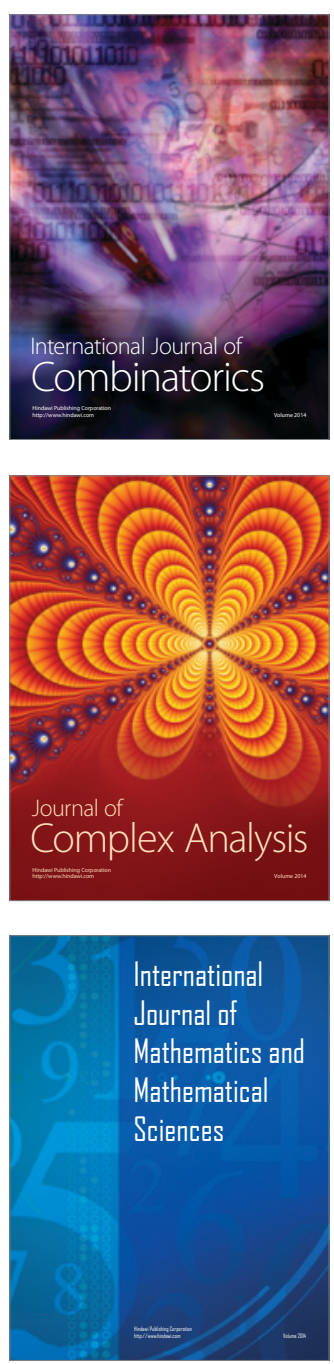
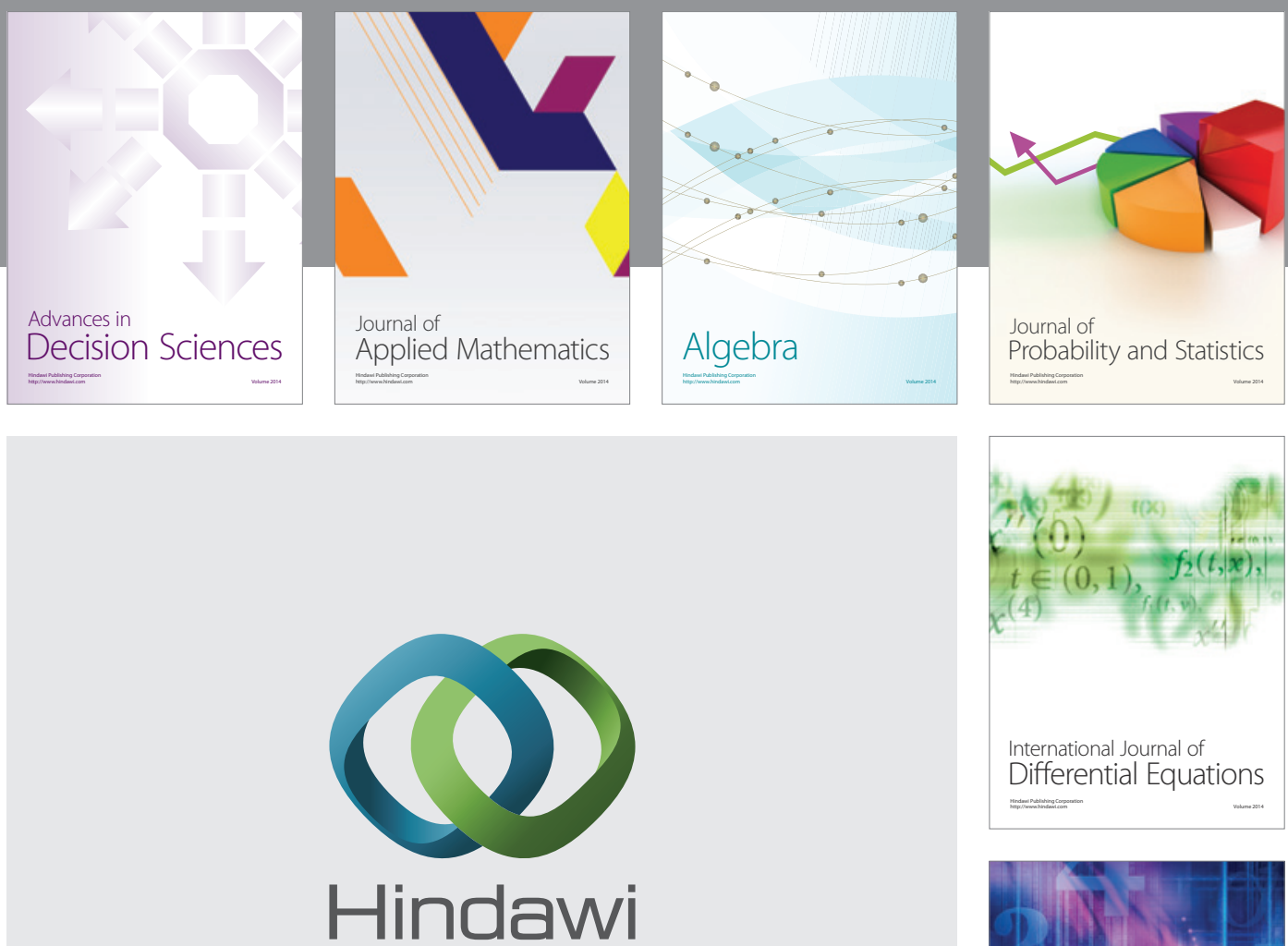

Submit your manuscripts at http://www.hindawi.com
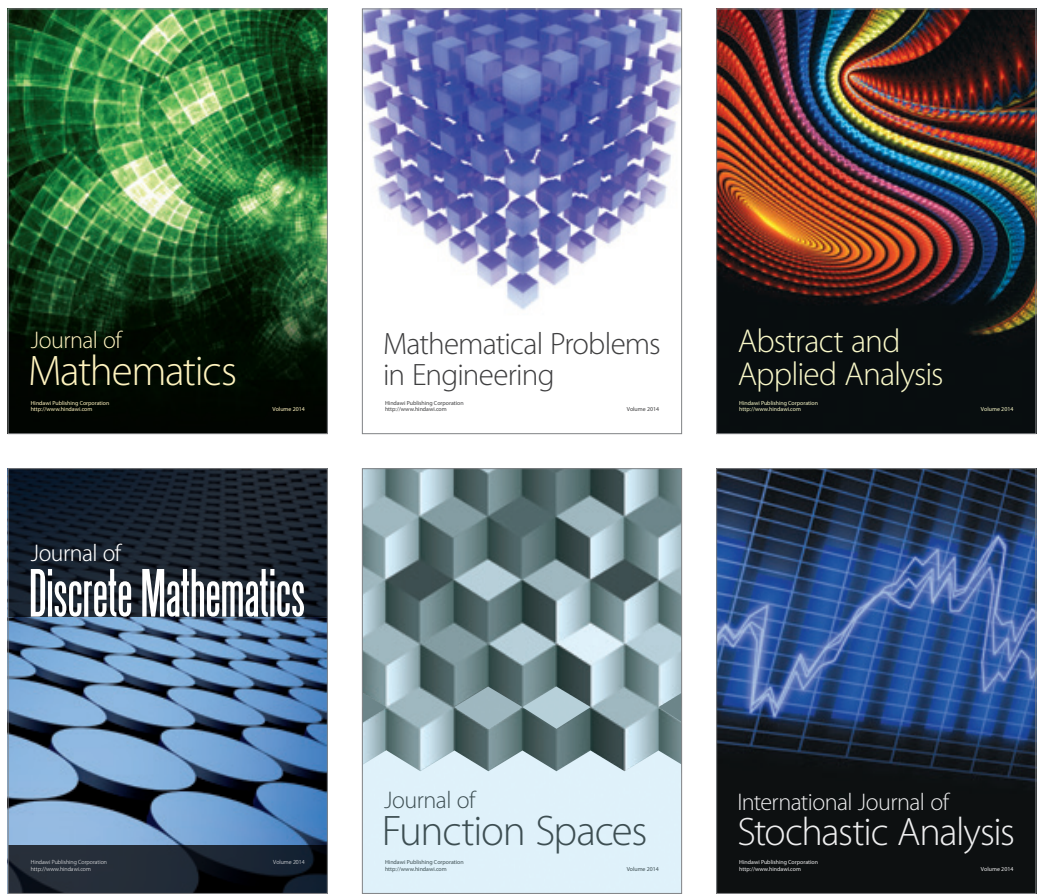

Journal of

Function Spaces

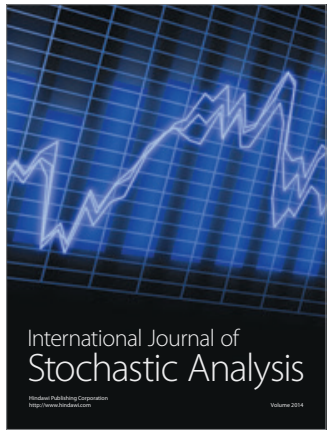

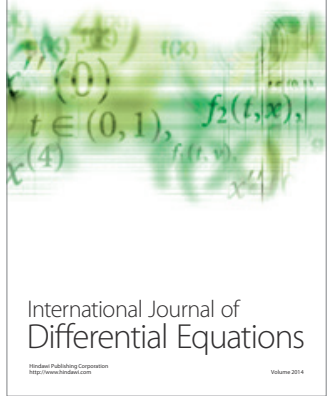
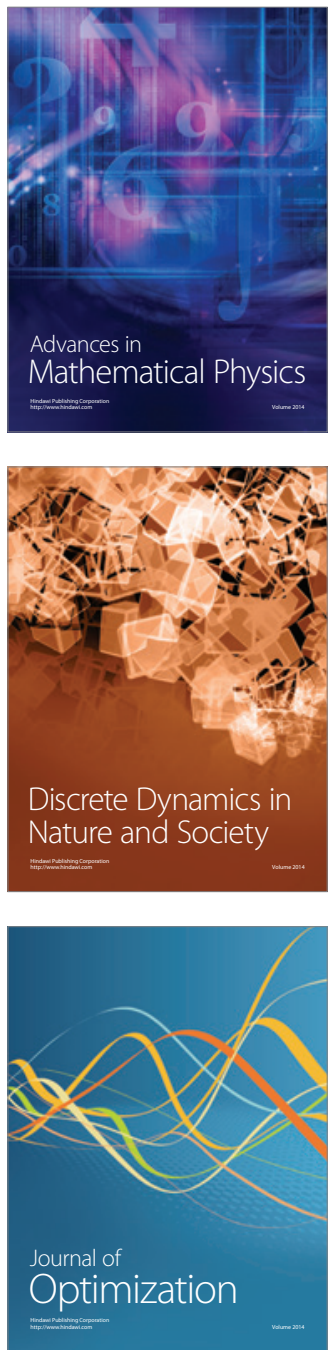